\title{
Was a cloud-cloud collision the trigger of the recent star formation in Serpens?
}

\author{
A. Duarte-Cabral ${ }^{1, \star}$, C. L. Dobbs $s^{2,3,4}$, N. Peretto ${ }^{1,5}$, and G. A. Fuller ${ }^{1}$ \\ 1 Jodrell Bank Centre for Astrophysics, School of Physics and Astronomy, University of Manchester, Oxford Road, Manchester, \\ M13 9PL, UK \\ e-mail: Ana.Cabral@postgrad.manchester.ac.uk \\ 2 School of Physics, University of Exeter, Exeter EX4 4QL, UK \\ 3 Max-Planck-Institut für extraterrestrische Physik, Giessenbachstraße, 85748 Garching, Germany \\ ${ }^{4}$ Universitäts-Sternwarte München, Scheinerstraße 1, 81679 München, Germany \\ ${ }^{5}$ Laboratoire AIM, CEA/DSM-CNRS-Université Paris Diderot, IRFU/Service d'Astrophysique, C.E. Saclay, Orme de merisiers, \\ 91191 Gif-sur-Yvette, France
}

Received 27 July 2010 / Accepted 10 January 2011

\section{ABSTRACT}

\begin{abstract}
Context. The complexity of the interstellar medium (ISM) is such that it is unlikely that star formation is initiated in the same way in all molecular clouds. While some clouds seem to collapse on their own, others may be triggered by an external event such as a cloud/flow collision forming a gravitationally unstable enhanced density layer.

Aims. This work tests cloud-cloud collisions as the triggering mechanism for star formation in the Serpens Main Cluster as has been suggested by previous work.

Methods. A set of smoothed particle hydrodynamics (SPH) simulations of the collision between two cylindrical clouds are performed and compared to (sub)millimetre observations of the Serpens Main Cluster

Results. A configuration was found that reproduces many of the observed characteristics of Serpens, including some of the main features of the peculiar velocity field. The evolution of the velocity with position throughout the model is similar to the observed one and the column density and masses within the modelled cloud agree with those measured for the SE sub-cluster. Furthermore, our results also show that an asymmetric collision provides the ingredients to reproduce lower density filaments perpendicular to the main structure, similar to those observed. In this scenario, the formation of the NW sub-cluster of Serpens can be reproduced only if there is a pre-existing marginally gravitationally unstable region at the time the collision occurs.

Conclusions. This work supports the interpretation that a collision between two clouds may have been the trigger of the most recent burst of star formation in Serpens. It not only explains the complicated velocity structure seen in the region, but also the temperature differences between the north (in "isolated" collapse) and the south (resulting from the shock between the clouds). In addition it provides an explanation for the sources in the south having a larger spread in age than those in the north.
\end{abstract}

Key words. stars: formation - stars: individual: Serpens - ISM: clouds - ISM: kinematics and dynamics - methods: numerical

\section{Introduction}

Observations of molecular clouds indicate that stars form in dense, clumpy filaments (e.g. André et al. 2010; Molinari et al. 2010). However, the processes controlling the formation of these structures and their role in triggering specific star-formation episodes are widely debated. One possible process could simply be a gravity-controlled one: quasi-static or dynamic gravitational collapse of non-spherical clouds can easily generate filamentary structures, which subsequently fragment to form stars (e.g. Palla \& Stahler 2002; Hartmann \& Burkert 2007). The contraction of an idealised isolated turbulent cloud leads to the formation of small-scale filaments ( $\sim 0.1 \mathrm{pc}$ long), within which stars begin to form, as shown by simulations from Bate et al. (2003); Klessen et al. (2005); Bate (2009a,b). Alternatively, external triggers can compress molecular gas to generate high-density fragmented filaments. These triggers can be of very different natures: ionisation/shock fronts around OB stars (e.g. Elmegreen \& Lada 1977; Dale et al. 2007), large-scale colliding flows generated by supernovae/galactic shear (e.g. Heitsch et al. 2008), or even molecular

\footnotetext{
* Funded by the Fundação para a Ciência e a Tecnologia (Portugal).
}

cloud collisions (e.g. Gittins et al. 2003; Kitsionas \& Whitworth 2007; Anathpindika 2009a,b).

Each of these processes have been tested with numerical simulations, and their respective efficiency in forming stars have been investigated. For some local clouds, we are beginning to acquire sufficiently detailed observations to generate a more informed picture of how star formation has progressed in those clouds. With these observations it becomes possible to perform dedicated simulations to model and better understand the processes at work. Only few such studies have been performed so far. For instance, Ballesteros-Paredes et al. (1999) used the results of their turbulent colliding flow simulations to argue that Taurus-Auriga formed through such a process; Peretto et al. (2007) used hydrodynamical simulations to model the global collapse and fragmentation of the NGC 2264-C protocluster; and Heitsch et al. (2009) compared flow-driven models for the formation of isolated molecular clouds with the Pipe nebula. In this paper, we use numerical simulations to investigate whether the recent star-formation episode observed in the Serpens Main Cluster could have been triggered by the collision of two molecular clouds, as proposed in Duarte-Cabral et al. (2010). 
Serpens is an interesting region in several aspects. It is a filamentary region with two compact young star-forming clumps. The gas emission reveals an interesting velocity structure and has an unusual temperature structure (Duarte-Cabral et al. 2010). The observations point to remnant signatures of the trigger behind the current young on-going star formation of Serpens. Duarte-Cabral et al. (2010) suggested that this region appears to have been affected by a collision of two clouds or flows. This scenario is also suggested by recent studies of the global dynamics of Serpens and other star-forming regions (e.g. Higuchi et al. 2010; Schneider et al. 2010; Galván-Madrid et al. 2010).

Here we use smoothed particle hydrodynamics (SPH) simulations of cloud-cloud collisions to attempt to reproduce the column density distribution and dynamical properties of the Serpens Main Cluster. A brief summary of the observationally inferred characteristics of Serpens and the motivation for the simulations are presented in Sect. 2. Section 3 explains the SPH code, the initial conditions and the adopted geometrical configurations. The results of the calculations are presented in Sect. 4. Section 5 presents a discussion of these results, where we compare the numerical results with the observations and choose the best-fit scenario to represent the region. The final remarks and conclusion are laid out in Sect. 6. Finally, in Appendix A we present temporal snapshots for all our models.

\section{The structure of the Serpens Main Cluster region}

The Serpens Main Cluster is a low-mass star-forming region at $260 \mathrm{pc}$ from the Sun (Straižys et al. 1996). It comprises two compact protoclusters, hereafter referred to as sub-clusters, lying in a 0.6 pc long filamentary structure along a NW-SE direction. At first sight, the NW and SE sub-clusters appear fairly similar. The dust emission (Davis et al. 1999, and Fig. 1) shows that they have similar masses within similar sized regions: $\sim 30 M_{\odot}$ in $0.025 \mathrm{pc}^{2}$ each, as estimated from the gas emission from $\mathrm{C}^{18} \mathrm{O}$ (Duarte-Cabral et al. 2010) and the $850 \mu \mathrm{m}$ continuum emission from SCUBA. Furthermore, both have sources at roughly the same stage of evolution, Class 0 and Class I protostars, which power a number of outflows (Davis et al. 1999; Graves et al. 2010).

Previous studies (Kaas et al. 2004; Harvey et al. 2006) have estimated an average age of $10^{5} \mathrm{yr}$ for these sources, which could possibly point to a triggering event. Older sources are also found in the field, but they appear more dispersed, without an obvious connection to the current protostars, and are no longer embedded or surrounded by any cold dust seen at submillimetre wavelengths. Presumably they belong to a different burst of starformation, which occurred 2 Myr before the current one, and these sources are now in the field as an unbound cluster.

In contrast to the similarities of the dust emission, the velocity structure and molecular emission from each sub-cluster are strikingly different. The NW is a well "behaved" sub-cluster with one main velocity throughout, matching the systemic velocity of the cloud with only a smooth velocity shift towards the edges of the sub-cluster. This is shown in the $\mathrm{C}^{18} \mathrm{O} J=1 \rightarrow 0$ positionvelocity (PV) diagram of Fig. 2 (top) and in Fig. 3, which shows the structure of the higher and lower velocity components of Serpens. In this NW sub-cluster, the gas and dust emission have similar distributions, with the gas emission peaks corresponding to the dust emission peaks. In addition, Spitzer $24 \mu \mathrm{m}$ sources are coincident with many of the $850 \mu \mathrm{m}$ sources (hereafter submillimetre sources; Fig. 1).

In contrast, the SE sub-cluster is quite different and its velocity structure is more complex. Optically thin tracers show

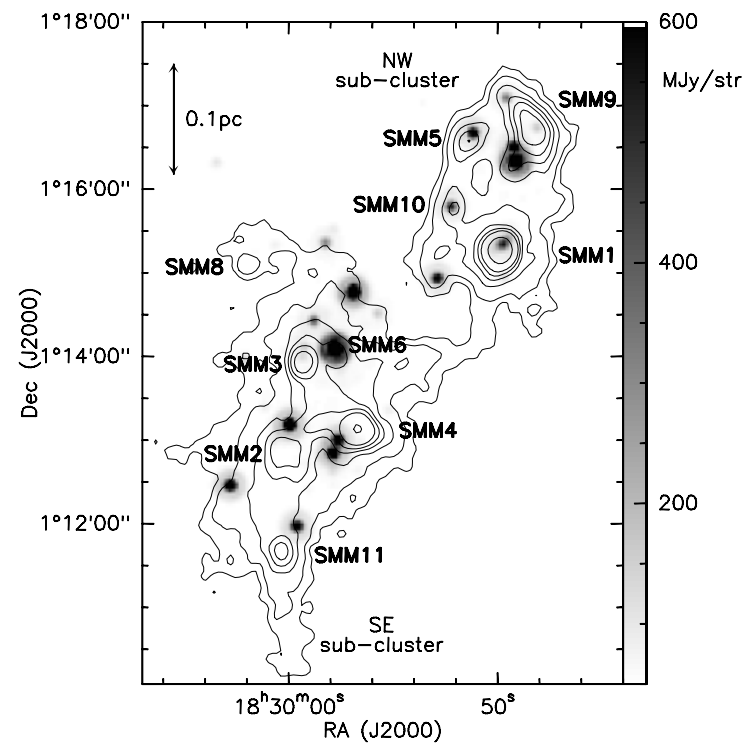

Fig. 1. Serpens region as seen in $850 \mu \mathrm{m}$ dust continuum emission with SCUBA (Davis et al. 1999) in contours, at 0.4, 0.6, 1, 1.4, 1.8, 2.4 and $5 \mathrm{Jy}$ beam $^{-1}$. In grey scale is the $24 \mu \mathrm{m}$ from Spitzer MIPS (Harvey et al. 2007). All sources seen in this image, both the $24 \mu \mathrm{m}$ and the $850 \mu \mathrm{m}$ sources, are Class 0 and I, with only a few flat-spectrum sources. The $850 \mu \mathrm{m}$ sources are labelled.
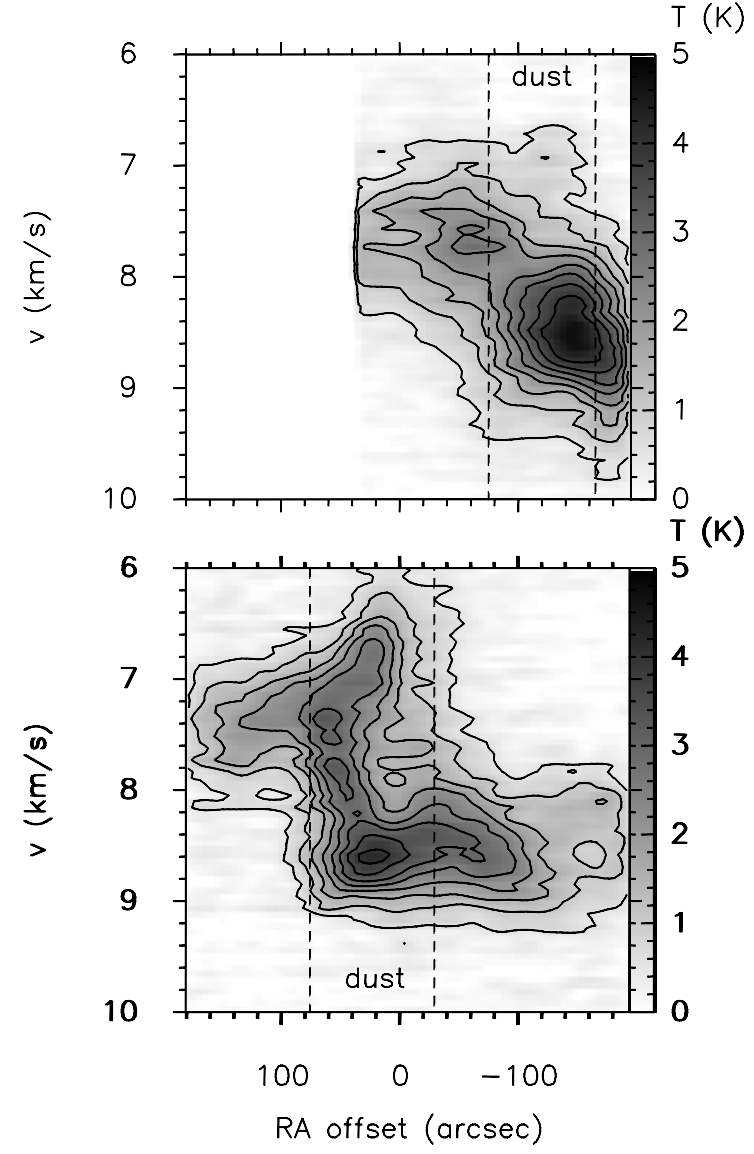

Fig. 2. Position-velocity diagrams at constant declination of $\mathrm{C}^{18} \mathrm{O} J=$ $1 \rightarrow 0$ (grey scale and contours) as examples of the typical velocity structure in the NW sub-cluster (top) and the SE sub-cluster (bottom) (Duarte-Cabral et al. 2010). Right ascension varies from $18^{\mathrm{h}} 30^{\mathrm{m}} 06^{\mathrm{s}}$ to $18^{\mathrm{h}} 29^{\mathrm{m}} 46^{\mathrm{s}}$ for all panels. Declinations are $1^{\circ} 16^{\prime} 48^{\prime \prime}$ (top panel) and $1^{\circ} 12^{\prime} 38^{\prime \prime}$ (lower panel). The positions of these cuts in the map are shown as white lines in Fig. 3. The dashed lanes labelled as "dust" represent the regions whose $850 \mu \mathrm{m}$ emission is above $0.6 \mathrm{Jy} \mathrm{beam}^{-1}$. 


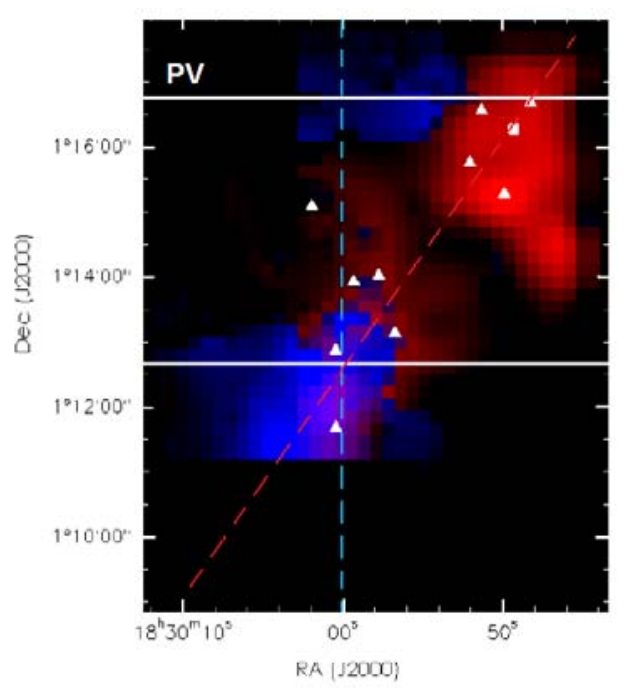

Fig. 3. $\mathrm{C}^{18} \mathrm{O} J=1 \rightarrow 0$ emission separated into blue and red components, using the line fitting and splitting by Duarte-Cabral et al. (2010). The blue emission corresponds to the total integrated intensity of the low-velocity cloud and the red emission corresponds to the highvelocity cloud. The submillimetre sources from the $850 \mu \mathrm{m}$ emission (Davis et al. 1999) are marked with white triangles and serve as a guide to the location of the dust emission. The positions of the PV diagrams in Fig. 2 are shown by the white solid lines, and the main axis of the blue-shifted and red-shifted cloud is shown as blue and red dashed line, respectively.

locations with a broad single component (in the north of the SE sub-cluster), which then splits into two clearly separated components (Fig. 3 and lower panel of Fig. 2). At the southern end of the sub-cluster, the filamentary structure is then only visible in one velocity component along the line of sight (lower PV diagram of Fig. 17 of Graves et al. 2010). Furthermore, the gas and submillimetre emission peaks do not coincide. Instead, the gas emission appears to peak between the submillimetre sources (Duarte-Cabral et al. 2010). Finally, there are only a few $24 \mu \mathrm{m}$ sources associated with the submillimetre dust continuum emission (Fig. 1). The overall picture resembles that of a region where star formation is an on-going process, with some younger sources (the purely submillimetre sources) and others older (the purely $24 \mu \mathrm{m}$ sources), unlike the NW sub-cluster where the sources appear to be all at the same evolutionary stage.

A final difference between the two Serpens sub-clusters is the gas temperature (Duarte-Cabral et al. 2010). The NW has a very homogeneous temperature around $10 \mathrm{~K}$, while the SE has higher and more varied temperatures, ranging between 10 and $20 \mathrm{~K}$. In addition, the temperature peaks are not coincident with any submillimetre source, but appear between them, in the region where the two velocity components overlap.

Thus the differences between the two sub-clusters indicate that they have had a complicated history. A scenario capable of explaining what triggered the Serpens star formation has to reproduce the inhomogeneities in the sources' age, velocity structure, and temperature distribution. The collision of two filamentlike clouds, colliding only over part of their length, could provide such a trigger.

\section{SPH simulations}

To test the proposal that the structure and star formation in the Serpens Main Cluster results from triggering by a cloud-cloud collision, we performed a set of SPH simulations, which are compared in detail with millimetre and submillimetre observations of Serpens.

\subsection{Numerical code}

The calculations were performed with an SPH code based on a version by Benz et al. (1990), which was subject to substantial modifications including sink particles (Bate 1995), variable smoothing lengths (Price \& Monaghan 2005), and magnetic fields (Price \& Bate 2007). The code has been frequently used for simulations of star formation (e.g. Dobbs et al. 2006; Bate 2009a).

We performed calculations that include the hydrodynamics and self-gravity, but not magnetic fields. We used sink particles, which are inserted in regions of high density $\left(10^{-12} \mathrm{~g} \mathrm{~cm}^{-3}\right)$ that are undergoing collapse, to represent protostars. However, for the whole analysis we used the frame of the simulation when the first sink particle appears, i.e. before stellar feedback is likely to have an effect. Therefore our results are not dependent on the details, or the dynamics of the sink particles.

For all calculations we adopted an isothermal equation of state. Again, we are only interested in the evolution of the clouds up to the point where star formation takes place. This renders a reasonable simplification given that we consider the structure of the gas prior to stellar feedback.

\subsection{Initial conditions}

Given the elongated appearance of the high- and low-velocity clouds in Serpens, we hypothesised that the velocity structure of the Serpens cluster is caused by the collision of two elongated clouds ( $\sim 1$ pc long). Cloud collisions have long been thought to be an important process in the ISM (since Oort 1954). They are potential triggers for star formation (e.g. Scoville et al. 1986; Vallee 1995), and are frequently found in galactic scale simulations (e.g. Dobbs 2008; Tasker \& Tan 2009). We do not suggest that cloud collisions are responsible for all star formation in the Galaxy, but aim to test how well this scenario can model the specific case of Serpens. Taking individual clouds from galactic simulations to model the collision is beyond the scope of this paper, and furthermore does not allow any freedom of the geometry of the clouds or collision. Instead, we assume a simplified initial configuration with cylindrical clouds for our models. The choice of cylinders is reasonable given that the most commonly observed aspect ratio of molecular clouds is $\sim 2$ (Koda et al. 2006). Thus our model is a simplified but plausible scenario in the local Galaxy.

For our calculations, we based the properties of the cylinders on the observations of Serpens, but with the requirement that the cylinders are not too far from virial equilibrium. We required the star formation to be primarily driven by the collision, and the cylinders should preferably retain their elongated shape as much as possible prior to the collision. Based on the observed spatial distribution of the Serpens' high- and low-velocity clouds, all simulations we ran involved two cylindrical clouds with radii of $0.25 \mathrm{pc}$. We kept one cylinder vertical and the other tilted throughout and, with the exception of run D (Table 1), the cylinders have a length of 0.75 and 1 pc respectively.

To ensure the stability of the cylinders prior to the collision, we used the formula for stability of finite cylinders given in Bastien (1983) to estimate the masses of the cylinders,

$J=\frac{G M f(L / D)}{L R_{\mathrm{g}} T / \mu}$, 
Table 1. Configuration of the different simulations.

\begin{tabular}{lcccc}
\hline \hline Run & Collision & LOS $\varangle \mathrm{V}$ & Length & Velocities \\
\hline $\mathrm{A}_{\text {non-T }}$ & Direct & $45^{\circ}$ & $1 \mathrm{pc}$ & Non-Turb \\
$\mathrm{A}_{\mathrm{T}}$ & Direct & $45^{\circ}$ & $1 \mathrm{pc}$ & Turb \\
$\mathrm{B}_{\text {non-T }}$ & Offset & $45^{\circ}$ & $1 \mathrm{pc}$ & Non-Turb \\
$\mathrm{B}_{\mathrm{T}}$ & Offset & $45^{\circ}$ & $1 \mathrm{pc}$ & Turb \\
$\mathrm{C}_{\mathrm{T}}$ & Offset & $0^{\circ}$ & $1 \mathrm{pc}$ & Turb \\
$\mathrm{D}_{\text {non-T }}$ & Offset & $0^{\circ}$ & $1.5 \mathrm{pc}$ & Non-Turb \\
\hline
\end{tabular}

where $R_{\mathrm{g}}$ is the gas constant, $G$ is the gravitational constant, $M$ is the mass of the cylinder, $L$ the length, $T$ the temperature and $\mu$ the mean molecular weight (we assumed $\mu=2.0$ for these calculations). $f(L / D)$ is a function measuring the shape of the cloud, but is of the order of unity for the dimensions of our cylinders. The cylinder is stable providing $J \lesssim 0.8$, a condition which reduces to approximately $M\left(M_{\odot}\right) / T(\mathrm{~K}) \lesssim 0.8$ for a 1 pc cylinder. In addition, we include an external pressure term, which is subtracted from each SPH particle when calculating pressure gradients (Dale et al. 2009). This represents a confining pressure from a low-density medium, which minimises diffusion of the outer parts of the cylinders, particularly in the runs including turbulence.

To mimic the supra-thermal line widths observed in Serpens, each model was also run for the same initial configuration adopting a turbulent velocity field for the particles. The turbulent field was set up according to the method of Bate et al. (2002), which is described in depth in Dubinski et al. (1995). The energy of the turbulent field $E(k)$ is chosen to follow a power law of $E(k) \propto k^{-4}$, with $k$ being the wavenumber. This gives, for a given scale, $r$, a velocity dispersion, $\sigma_{v}$, such that $\sigma_{v} \propto r^{0.5}$. This is similar to the observed Larson relations for clouds (Larson 1981), although we do not aim to capture the precise details of the internal motion, but rather to compare results with and without turbulence.

From the velocity-space distribution of $\mathrm{C}^{18} \mathrm{O}$ emission in the Serpens Main Cluster (see Fig. 3 and Duarte-Cabral et al. 2010), we found that the higher velocity component (red in Fig. 3 ) is tilted at $55^{\circ}$ angle from the horizontal plane, and is situated mostly to the east on the map. The lower velocity component (blue in Fig. 3) is roughly vertical and in the west of the map (overlapping only along the SE end of the filament of star formation). To match the observations, the higher velocity component corresponds to the longer and tilted cylinder moving away from us on the right-hand side (see Fig. 4 red cylinder). This cylinder has an elevation angle of $55^{\circ}$ above the horizontal plane and an azimuth angle of $0^{\circ}$, i.e. the axis is in a plane parallel to the $y^{\prime} z^{\prime}$ plane. The lower velocity component corresponds to the vertical cylinder, which is coming towards us from the left-hand side (see Fig. 4 blue cylinder).

The velocities of the cylinders are chosen to agree with the velocity difference of the gas in the Serpens cluster (see lower panel in Fig. 2), which would correspond to the velocities of the clouds during the collision. The initial velocities of each cylinder are $v= \pm 1 \mathrm{~km} \mathrm{~s}^{-1}$, i.e. $v_{x^{\prime}}$ and $v_{y^{\prime}}= \pm 0.77 \mathrm{~km} \mathrm{~s}^{-1}$. The cylinders have no initial net velocity in the $z$ direction (and without turbulence the particles have zero velocity in the $z$ direction).

Each of the calculations were run with and without a turbulent velocity field. The differences between the four runs are sketched in Fig. 4 and summarised in Table 1, where "collision" specifies if the collision is off-centred or head-on with respect to the centre of the cylinders; "LOS $\varangle \mathrm{V}$ " represents the angle between the line of sight (LOS) and the direction of the cylinders' motion; "length" is the height of the longer (i.e. the tilted) cylinder in each run; "velocities" specify the initial distribution of velocities: "non-turb" means zero velocity dispersion in the initial conditions, and "turb" indicates a turbulence-generated velocity dispersion amplitude of $0.5 \mathrm{~km} \mathrm{~s}^{-1}$ (in each direction). This is similar to the observed turbulent velocity dispersion in Serpens, which is of the order of $0.5 \mathrm{~km} \mathrm{~s}^{-1}$ (Duarte-Cabral et al. 2010)

All other properties of the cylinders, such as the temperatures and densities, are fixed. In the "short-cylinder" runs (A, B, and C) we used 500000 particles in total, with 250000 particles in each cylinder. Initially, the particles are uniformly randomly distributed to provide a roughly uniform density distribution within each cylinder.

As discussed in Duarte-Cabral et al. (2010), the NW subcluster tends to have a uniform temperature of 10-13 K. On the other hand, the temperature of the SE sub-cluster is more difficult to estimate, given that there are two clumps along the line of sight. Duarte-Cabral et al. (2010) obtained gas temperatures between 10 and $20 \mathrm{~K}$. However, as shown by Eq. (1), a kinetic temperature of $30 \mathrm{~K}$ is required for a $30 M_{\odot}$ cylinder to be relatively stable. Hence we decided to add this extra internal pressure in the simulations in order to avoid the collapse of the cylinders before the collision. We chose masses of 30 and $45 M_{\odot}$ for the two cylinders, and verified that for a temperature of $30 \mathrm{~K}$ the cylinders did not collapse prior to the collision. This does not have any impact on the velocity and column density structure discussed in the rest of the paper.

We set the cylinders to collide at an angle of $45^{\circ}$ with regard to the line of sight. In addition, we chose to position the cylinders so that either they collided head on (run A), or that their major axis were offset by $0.25 \mathrm{pc}$ (run B). In the direct collision, model $\mathrm{A}$, we centred one cylinder at cartesian coordinates $(0,0,0) \mathrm{pc}$ and the second (longer) cylinder at $(0.8,0.8,0) \mathrm{pc}$ (Fig. 4 top left panel). For the offset collision, model B, the second cylinder was instead placed at $(0.8,1.05,0) \mathrm{pc}$. We illustrate the offset configuration in Fig. 4 (top right panel).

The results from runs A and B are summarised in Sects. 4.1.1 to 4.1.2. These runs show that by the time sink particles are formed, the two cylinders fully overlap along the line of sight, even though they did not interact everywhere. This means that we can see two velocity components co-existing in most regions (e.g. Fig. 8 top left panel), which is inconsistent with the observations, where the two velocity components overlap only where the collision takes place.

Therefore, we changed the perspective so that the motion of the cylinders is purely along the line of sight, model C (Fig. 4 bottom left panel), to restrict the region where the two components overlap to where the cylinders interact directly. Finally, model D (Fig. 4 bottom right panel) aims to reproduce the whole extent of the observed cloud and, therefore, it is similar to model $\mathrm{C}$, except that the cylinders were made one and half times longer by extending the top and bottom of the cylinders. The number of particles was correspondingly increased in this calculation.

\section{Results}

As described in Sect. 2, the Serpens Main cluster is divided into two sub-clusters, the SE and NW sub-clusters. All observational evidence tends to show that the SE sub-cluster is at the interface of a cloud-cloud collision, as opposed to the much more quiescent NW sub-cluster. For this reason, in the cloud-cloud collision simulations we present here, we first attempted to reproduce the physical properties of the SE sub-cluster. However, given the obvious physical connection between these two sub-clusters, we 
A. Duarte-Cabral et al.: Was a cloud-cloud collision the trigger of the recent star formation in Serpens?

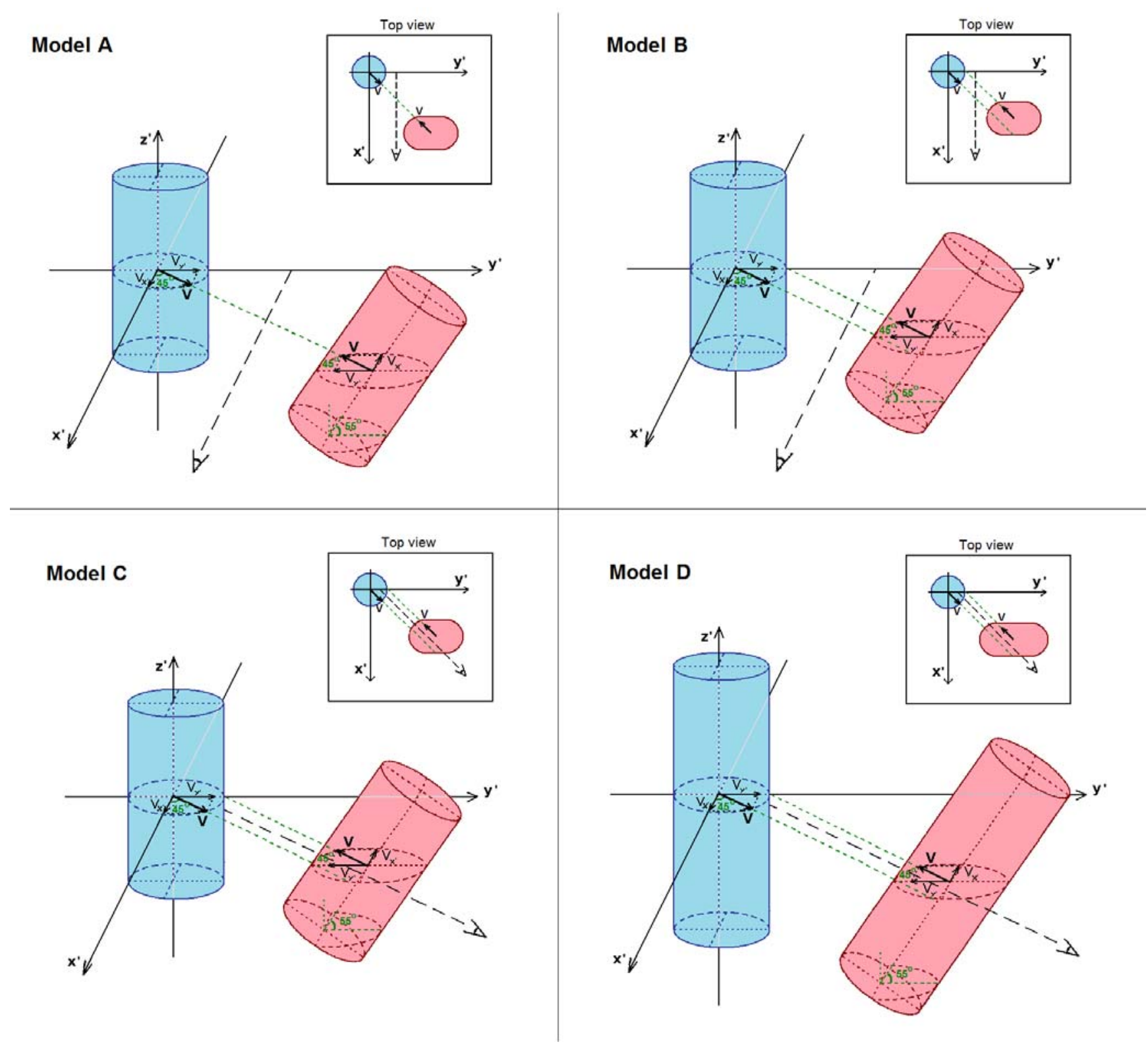

Fig. 4. Diagrams showing the starting configuration of the cylinders for the direct collision (model A, top left panel), offset collision (model B, top right panel; and model $\mathrm{C}$, lower left panel) and offset collision with longer cylinders (model D, lower right panel). The black dashed line represents the line of sight, and the green dashed lines are the direction of the motion of the cylinders. On the top right corner of each panel is a sketch with the configuration as viewed from the top.

also tried in a second set of simulations to trigger the formation of a NW-like sub-cluster as a by-product of the collision.

To compare the velocity structure of the simulations we will use the observational data and results from Duarte-Cabral et al. (2010): IRAM $30 \mathrm{~m}$ telescope observations of $\mathrm{C}^{18} \mathrm{O} J=1 \rightarrow 0$. We also refer to the analysis of Serpens molecular data from the JCMT GBS HARP data of $\mathrm{C}^{18} \mathrm{O} J=3 \rightarrow 2$ (Graves et al. 2010). For $\mathrm{H}_{2}$ column density comparisons, we used the SCUBA $850 \mu$ m emission from JCMT (Davis et al. 1999).

The comparison of the simulations with the observations is primarily focused on observational characteristics of the Serpens SE sub-cluster because this is the region that appears to be directly influenced by the collision. However, even in the shortcylinder runs that do not form the NW sub-cluster, some of the velocity characteristics of the entire cloud are also taken into account. Thus, the following characteristics are the main focus points for a comparison:
- Column density structure: an elongated/filamentary shape aligned in a NW-SE direction, sub-clumped (Fig. 1).

- The mass and size of the southern clump compared with those of the denser parts of the SE sub-cluster $\left(\sim 30 M_{\odot}\right.$ within $\sim 0.025 \mathrm{pc}^{2}$, measured using the $850 \mu \mathrm{m}$ emission above $190 \mathrm{mJy}^{-1}$ beam $^{-1}$, which corresponds to column densities above $0.1 \mathrm{~g} \mathrm{~cm}^{-2}$ at a temperature of $10 \mathrm{~K}$ ).

- Overall velocity structure: a single red-shifted component in the north, with a fainter blue component spatially offset to the east (top panel of Fig. 2 top panels and Fig. 3). Double (overlapping) components in the south, where the sources are being formed (Fig. 2 lower panel and Fig. 3) with a gradient from east to west of increasing velocities, up to $1.5 \mathrm{~km} \mathrm{~s}^{-1}$ (Fig. 13). Moving further south, this velocity structure should evolve back into one component along the less dense gas of the filament (Fig. 13, and lower PV diagram of Fig. 17 of Graves et al. 2010). 
- Velocity structure of the less dense material: thin eastern filaments perpendicular to the main filament of Serpens (visible in blue and green on the left-hand side of the main filament in Fig. 13).

In order to compare our results with the observations, we first constructed a datacube from the simulated 3D cloud collisions. For each simulation, we define the $x$ direction as the line of sight, so that the plane of the sky is the $y z$ plane. Choosing the timestep of the simulations where the first sink particle is formed, we created a datacube of column density for a space-space-velocity $3 \mathrm{D}$ grid. In the spatial planes we convolved the models with a Gaussian with a FWHM of 10 pix $(0.02$ pc), which corresponds to the $22^{\prime \prime}$ spatial resolution of the IRAM-30 m telescope observations of $\mathrm{C}^{18} \mathrm{O} J=1 \rightarrow 0$ at the distance of Serpens. To reproduce the thermal velocity dispersion of Serpens, the velocity space was convolved with a normalised Gaussian of $0.4 \mathrm{~km} \mathrm{~s}^{-1}$ $F W H M$, corresponding to the thermal line width of $\mathrm{H}_{2}$ at $10 \mathrm{~K}$.

Gas temperature, density, and abundance all affect the relation between the true column density of a cloud and the emission seen in a molecular line. However, as discussed in Duarte-Cabral et al. (2010) and Graves et al. (2010), in Serpens the $\mathrm{C}^{18} \mathrm{O}$ appears to be a faithful tracer of the overall velocity structure of the cloud and does not appear to be significantly affected by outflow or infall motions, while the $850 \mu \mathrm{m}$ emission traces the global mass distribution in the region. To assess the success of the simulations in modelling Serpens, we therefore compare the models with the velocity structure of the $\mathrm{C}^{18} \mathrm{O}$ and the overall column density distribution from the dust emission.

\subsection{Modelling the Serpens SE sub-cluster}

We initially tried to reproduce the characteristics of the SE subcluster with the short-cylinder calculations: runs A, B, and C. In this section, we describe the results of these simulations. Taking the best-fit configuration for the short-cylinders, we then performed simulations with more extended cylinders that are designed to reproduce the whole cloud (Sect. 4.2).

\subsubsection{Non-turbulent runs: $A_{n o n-T}$ and $B_{n o n-T}$}

Fundamentally, the only difference between these two simulations is that for $A_{n o n-T}$ the centres of gravity of the cylinders are colliding head-on, maximising the loss of kinetic energy in the shock, while for $\mathrm{B}_{\text {non- } \mathrm{T}}$ the centres of gravity are shifted and therefore the collision becomes softer, with the gas being able to conserve more of its initial kinetic energy. While the first configuration is a very peculiar one, the second one is very general, corresponding to the most likely situations of cloud-cloud collision. As a result, the formation of sink particles in $B_{n o n-T}$ is slower than in $\mathrm{A}_{\text {non- } \mathrm{T}}$, and while it occurs only at the interface region for the latter, in $B_{n o n-T}$ sink particles are more widely distributed, along a dense filament connecting both clouds (see Fig. 5 and Appendix A, Fig. A.1).

The most significant difference between the results of these two simulations is the velocity structure at the collision interface. In $A_{n o n-T}$, three distinct velocity components are present: the two velocities of the individual cylinder, plus an intermediate velocity of the shocked interface gas (Fig. 6 left panel). In $\mathrm{B}_{\text {non- } \mathrm{T}}$, the velocity structure is continuously connecting the initial velocities of the cylinders (Fig. 6 right panel). Thus, the offset collision (B) is better able to reproduce the observations (Fig. 2).

\subsubsection{Turbulent runs: $A_{T}$ and $B_{T}$}

In these simulations the net effect of turbulence is to render the cylinders inhomogeneous in density and velocity, making them more realistic-looking clouds. One of the important features we see in Fig. 7 (and Appendix A, Fig. A.2), are the channels of material perpendicular to the main filament. These are reminiscent of features often observed in molecular clouds (e.g. Myers 2009). These features will be further discussed in Sect. 5. Another important aspect of injecting turbulence is that is generates density seeds. Before the first sink particles begin to form, we see several regions where the density is beginning to grow. As soon as one of these regions is sufficiently dense, it will attract the remaining less dense sub-clumps to form a major single structure.

At the end of both runs, the material at a column density greater than $0.1 \mathrm{~g} \mathrm{~cm}^{-2}$ is distributed over similar sized regions to the non-turbulent models, $0.025 \mathrm{pc}^{2}$ and $0.032 \mathrm{pc}^{2}$, for runs $\mathrm{A}_{\mathrm{T}}$ and $\mathrm{B}_{\mathrm{T}}$ respectively. For $\mathrm{A}_{\mathrm{T}}$, a total of $29 M_{\odot}$ is in these high column density regions (39\% of the total mass of the cylinders), while for $\mathrm{B}_{\mathrm{T}}$ the mass is $34 M_{\odot}(45 \%$ of the total mass).

In terms of the velocity structure, the direct collision model, $\mathrm{A}_{\mathrm{T}}$, still produces too many distinct velocity components without smooth trends (Fig. 8 left panels), for the same reasons as for the $\mathrm{A}_{\text {non- } \mathrm{T}}$ model. On the other hand, the $\mathrm{B}_{\mathrm{T}}$ model produces a very interesting velocity structure. To the north (positive $z$ ) we mainly see the tilted cylinder and begin to detect the vertical cylinder on the left-hand side. The transition between the two is not sharp nor double-peaked, but a fairly broad smooth transition (Fig. 8 top right panel). A well defined double-peak structure is only detected in the central region of the model, where sink particles have formed (Fig. 8 middle right panel). South of this region, the column density becomes again dominated by one component from the tilted cylinder travelling away from us (Fig. 8 bottom right panel), as also found towards the south end of the Serpens filament by Graves et al. (2010). The results from this run $\left(\mathrm{B}_{\mathrm{T}}\right)$ are more consistent with the observations of the SE subcluster of Serpens (Sect. 2), but the change in velocity across the cloud is smaller than the observed one $\left(\sim 0.5 \mathrm{~km} \mathrm{~s}^{-1}\right.$ instead of $\sim 1 \mathrm{~km} \mathrm{~s}^{-1}$ ).

We also ran a model with an intermediate turbulence level (not shown) with a velocity dispersion of $0.3 \mathrm{~km} \mathrm{~s}^{-1}$. This model showed that the geometric configuration is the dominant factor in determining the main velocity characteristics of the resulting cloud, while the density distribution is sensitive to the level of turbulence. Lower levels of turbulence result in a more filamentary structure, with more sink particles forming along the denser filament. Increased values of initial turbulence tend to disrupt the distribution of cloud's material, inhibiting the formation of further clumps.

\subsubsection{Line of sight, short cylinder run: $\mathrm{C}_{\mathrm{T}}$}

Model $\mathrm{C}_{\mathrm{T}}$ is simply a re-projection of model $\mathrm{B}_{\mathrm{T}}$ so that it is viewed along the axis of the relative motion of the cylinders. The time evolution of $\mathrm{C}_{\mathrm{T}}$ is shown in Appendix A, Fig. A.3.

From this perspective (Fig. 9) the low-density material is less filamentary and more spatially extended, because the parts of the cylinders that do not interact, do not overlap along the line of sight any longer. Therefore, the extent of the lower density material is greater to the north, where the cylinders do not merge, than in the south (negative $z$ ). In this projection, the material at high column density above $0.1 \mathrm{~g} \mathrm{~cm}^{-2}$ covers a region of $0.02 \mathrm{pc}^{2}$ in area with a mass of $22 M_{\odot}$. 
A. Duarte-Cabral et al.: Was a cloud-cloud collision the trigger of the recent star formation in Serpens?

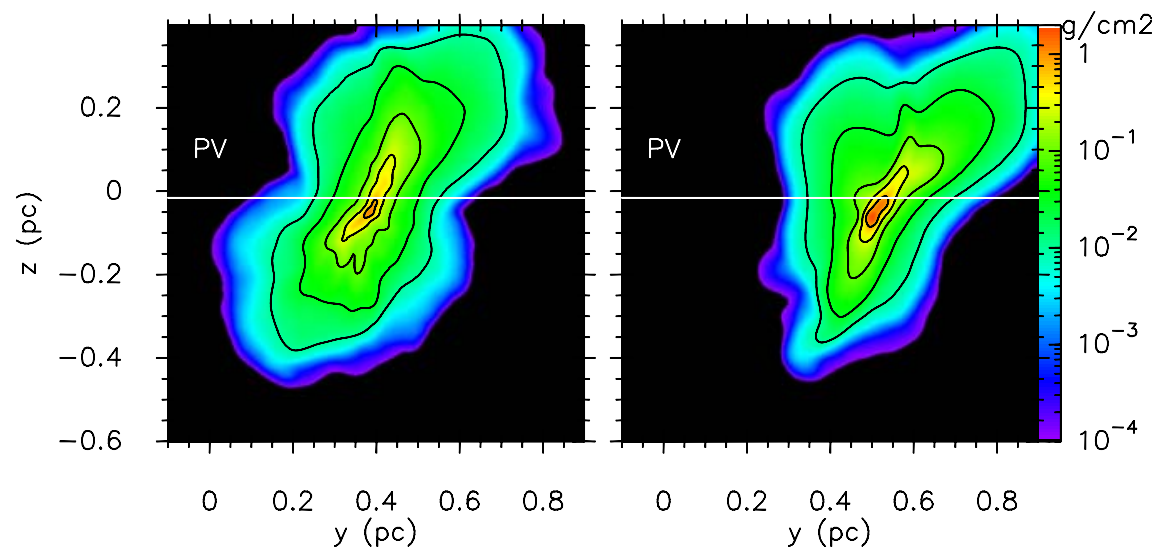

Fig. 5. Colour scale and contours of the total column density along the line of sight for the non-turbulent runs: the centred collision, $A_{\text {non-T }}$, on the left; and off-centred collision, $\mathrm{B}_{\text {non-T }}$, on the right. The contour levels are $0.01,0.03,0.1,0.3$ and $0.5 \mathrm{~g} \mathrm{~cm}^{-2}$ for both figures. The white horizontal lines show the cuts for the PV diagrams in Fig. 6.
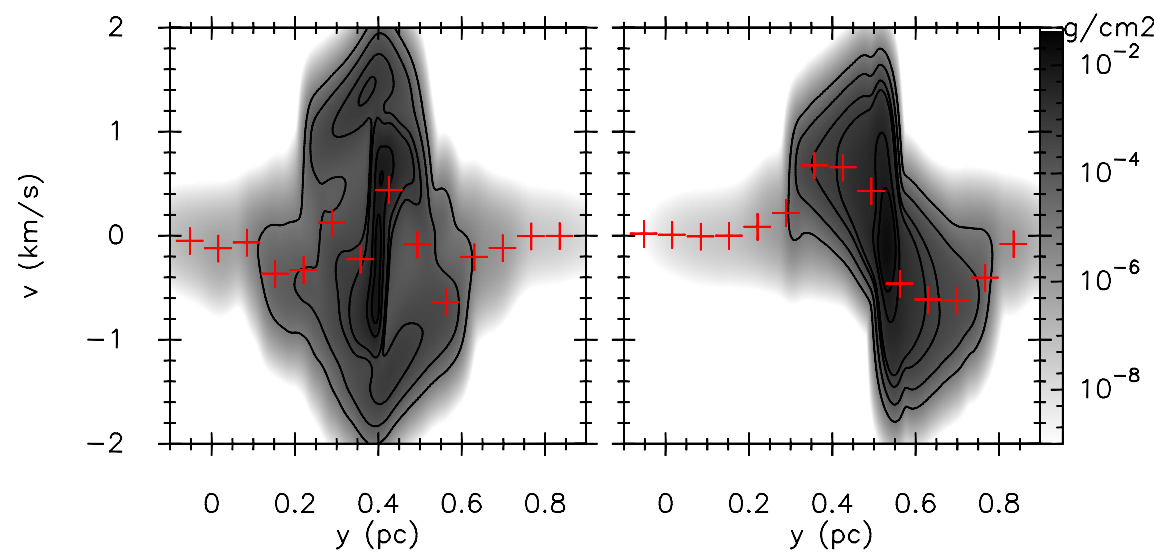

Fig. 6. Position-velocity diagram at $z=-0.02 \mathrm{pc}$ (colour scale and contours) for the non-turbulent direct and offset runs, $\mathrm{A}_{\text {non-T }}$ and $\mathrm{B}_{\text {non-T }}$, left and right respectively. The displayed velocity corresponds to the component along the line of sight. Contours at $10^{-5}, 10^{-4}, 5 \times 10^{-4}, 10^{-3}, 5 \times 10^{-3}$, $10^{-2}$ and $3 \times 10^{-2} \mathrm{~g} \mathrm{~cm}^{-2}$. The red crosses represent the column density weighted velocities and are plotted as an auxiliary tool to see the velocity changes along the diagram. Note that the velocities from the direct collision (left) in the central region seem to be more complex than those observed (Fig. 2 lower panel), whereas those from the offset collision (right) show a smooth shift in velocities from one component to another, likely because in the offset collision we see the parts of the clouds that do not collide more clearly.

The general shape and trend of the velocity structure (Fig. 10), although slightly more complex, is similar to that seen in $\mathrm{B}_{\mathrm{T}}$ and consistent with the velocity trend seen in the observational PV diagrams. Overall, this run $(C)$ reproduces Serpens better than run $\mathrm{B}$, because it now shows a more evident velocity change across the cloud.

\subsection{Representation of both SE and NW Serpens sub-clusters}

\subsubsection{Line of sight, long cylinders, run: $D_{\text {non-T }}$}

Overall, model $\mathrm{C}_{\mathrm{T}}$ satisfactorily reproduces the observations of the SE sub-cluster. However, it does not have sufficient Jeans masses to form a second sub-cluster comparable to the NW subcluster in Serpens, even though the velocity structure of the simulation is very similar to the observed one. Therefore, we increased the length and mass of the cylinders to investigate the possible formation of a separate structure in the north, where the cylinders do not interact. If the non-interacting northern region is massive enough to be close to unstable, the collision in the south of the cylinders may induce its rapid collapse without greatly affecting its systemic velocity.
A non-turbulent model with $\sim 1.5$ pc long cylinders, colliding off-centre and along the light of sight (model $\mathrm{D}_{\text {non-T }}$ ), does indeed form two sub-clusters. The collision in the south leads to the formation of a sink particle in the south, i.e. the synthetic SE sub-cluster. Later on, a sink particle is formed in the north of the tilted cylinder, along the cylinder axis (time evolution in Appendix A, Fig. A.4). Finally, more sink particles are formed in the south, as the vertical cylinder crosses through the tilted one (Fig. 11). At the end of this run, when sink particles are formed throughout the clouds, the column density distribution is much more filamentary than for the short cylinder calculation. There are two visible sub-clusters: one in the NW and one in the SE. The total mass in these sub-clusters amounts to $57 M_{\odot}$, $52 \%$ of the total mass in this case, distributed over an area of $0.035 \mathrm{pc}^{2}$. However, the relative size and mass of each individual sub-cluster is not quite as similar as seen in Serpens. The simulated SE sub-cluster has a mass of about $30 M_{\odot}$ in an area of $\sim 0.015 \mathrm{pc}^{2}$, whereas the NW sub-cluster contains $12 M_{\odot}$ in a similar area.

The formation of a sink particle in the north occurs at $8 \times 10^{5} \mathrm{yr}$ and is mainly due to the initial instability of the cylinder itself. As a test, we ran a simulation with only an isolated 

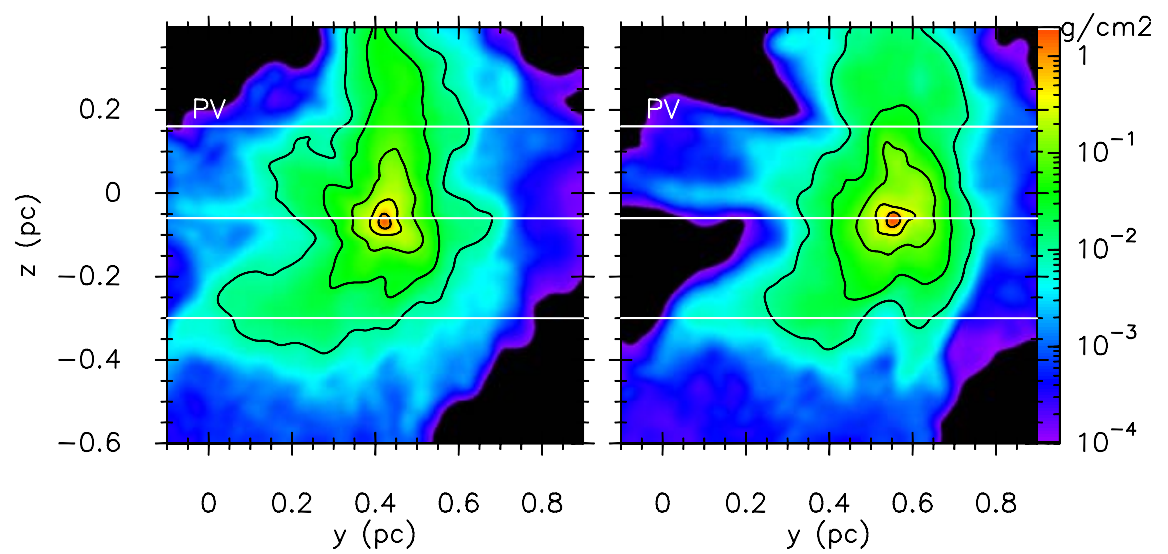

Fig. 7. Colour scale and contours representing the total column density along the line of sight for the turbulent runs: the centred collision, $\mathrm{A}_{\mathrm{T}}$, on the left; and off-centred collision, $\mathrm{B}_{\mathrm{T}}$, on the right. The contour levels are as in Fig. 5. The white horizontal lines show the cuts for the PV diagrams in Fig. 8. Both turbulent runs show a final cloud which is much more structured and fits the observations better than Fig. 5.

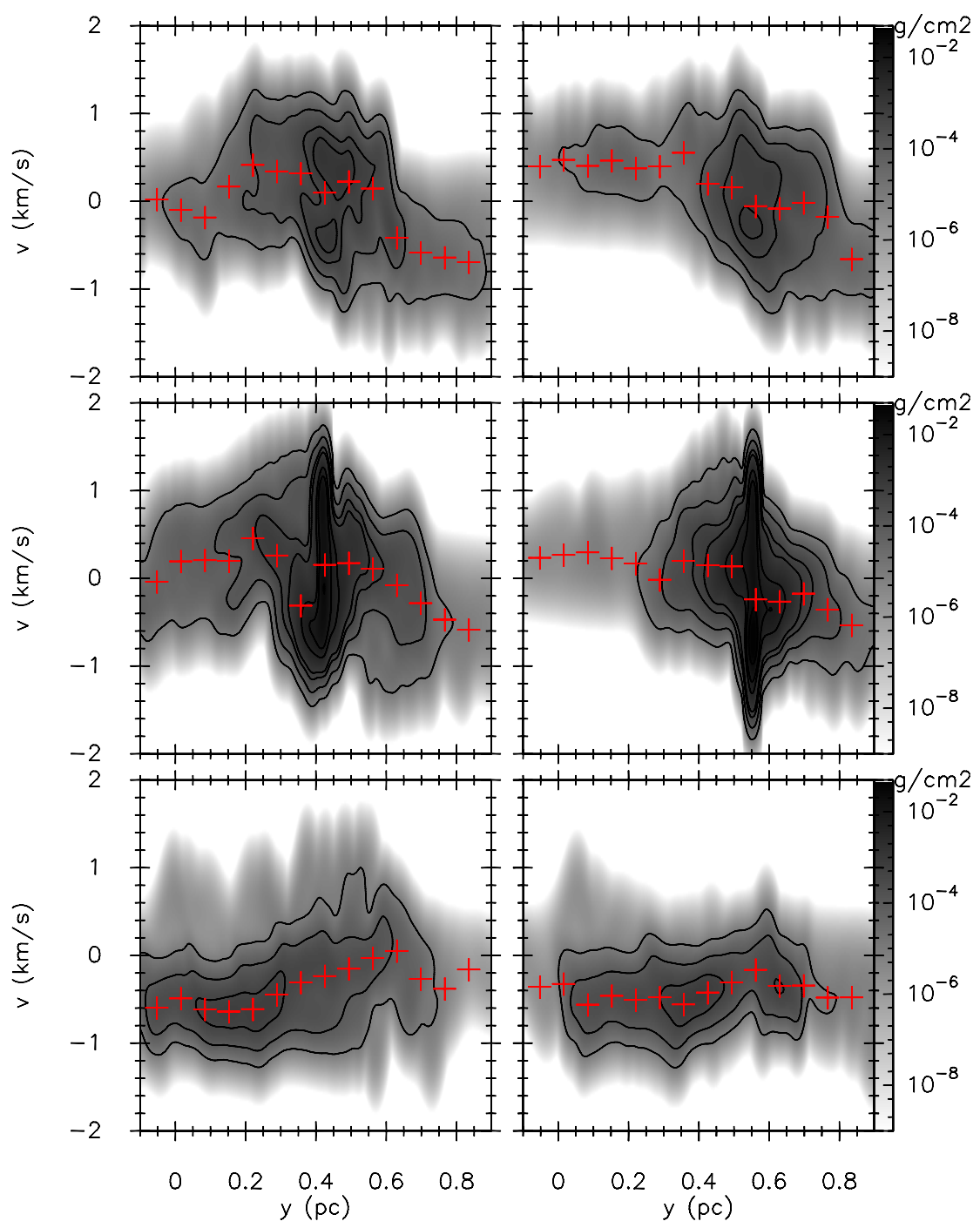

Fig. 8. Position-velocity diagrams in colour scale and contours, at $z=0.16 \mathrm{pc}($ top $), z=-0.06 \mathrm{pc}$ (middle) and $z=-0.30 \mathrm{pc}($ bottom) for the turbulent direct and offset runs, $\mathrm{A}_{\mathrm{T}}$ and $\mathrm{B}_{\mathrm{T}}$, left and right respectively. The contour levels and red crosses are as in Fig. 6. As expected from Fig. 6, the velocities are more complex in the A run than the observed ones. The top panels show that the direct collision (left) produces two velocity components in the northern part of the cloud, whereas the offset collision B (right) and the observations only show one (Fig. 2). From the middle and lower panels, the differences are less obvious, but the middle panel shows that the direct collision exhibits a more "messy" velocity structure rather than a clear transition from high to low velocities. 
A. Duarte-Cabral et al.: Was a cloud-cloud collision the trigger of the recent star formation in Serpens?

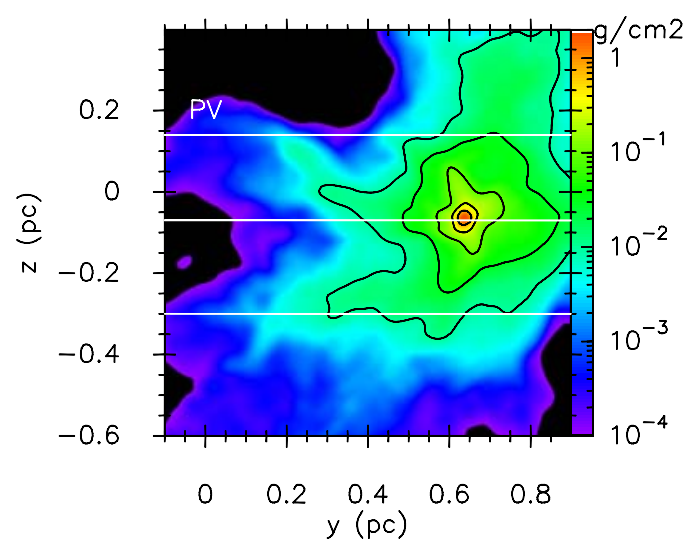

Fig. 9. Total column density for the purely along the line of sight turbulent run, $\mathrm{C}_{\mathrm{T}}$. Unlike runs $\mathrm{A}$ and $\mathrm{B}$, we find a low-density region in the north (large $z$ ), because with this orientation we see regions that have not collided. The contour levels are as in Fig. 5.

non-turbulent cylinder, with the same mass and size as the tilted one. This simulation showed that it would form sink particles along the cylinder axis at $10^{6} \mathrm{yr}$. Therefore, the collision in the south does not trigger the formation of this sink particle in the north, but only speeds up its collapse.

Interestingly, several different turbulent runs with this configuration failed to induce the collapse of any structure in the north. The collapse only occurs with extremely low levels of turbulence (with amplitude of $\sim 0.05 \mathrm{~km} \mathrm{~s}^{-1}$ ), almost indistinguishable from the non-turbulent run. This is due to the turbulent velocities that cause the cloud to disperse from its initial configuration and become less gravitationally bound than in the absence of turbulence. From this, we conclude that the effect of the collision felt by the north region is quite subtle, and would only have an influence on the collapse there if the region is almost gravitationally unstable prior to the effect of any external perturbation.

The discrepancy in the relative masses of the two subclusters in the simulation compared to Serpens, and indeed the failure to form two sub-clusters in the turbulent simulations could be addressed by relaxing the over-simplistic uniform conditions in our initial conditions. Both the density distribution and turbulent velocity distributions are likely to be more inhomogeneous in real colliding clouds than in our models. This discrepancy could also be suggesting that we are missing a physical ingredient such as magnetic pressure, which could help to maintain the filamentary shape of the clouds even with a high level of turbulence. However, we refrained from adding this additional complexity to the models since the nature of the inhomogeneities is poorly constrained and unlikely to provide any further significant physical insight into the processes in this region.

In terms of its spatial evolution, the velocity structure of the $D_{\text {non-T }}$ is similar to the observed one. From Fig. 12 we see as we move from north to south (i.e. from positive $z$ to negative z) two spatially separated components, which then overlap where the collision takes place, to finally end-up with a single velocity component in the south. These PV diagrams can be compared with the more detailed PV diagrams across the entire cloud from Duarte-Cabral et al. (2010) and Graves et al. (2010). Also note that the column densities are higher at velocities corresponding to the tilted cylinder. An increase in the density of the tilted cylinder to produce a more massive NW sub-cluster would increase the difference in column densities between the

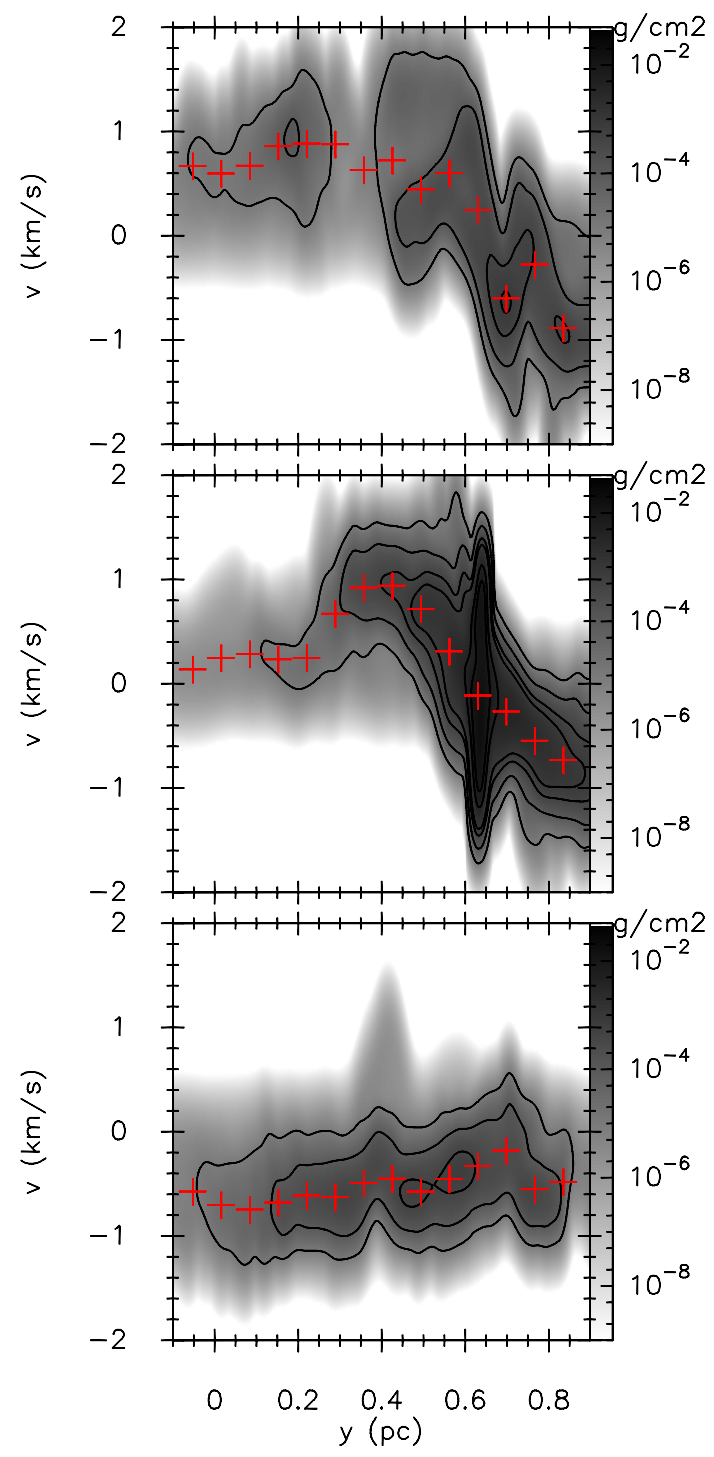

Fig. 10. Position-velocity diagram in colour scale and contours, at $z=$ $0.14 \mathrm{pc}$ (top), $z=-0.07 \mathrm{pc}$ (middle) and $z=-0.30 \mathrm{pc}$ (bottom), for the turbulent, line of sight, short cylinders run $\mathrm{C}_{\mathrm{T}}$. The contour levels and red crosses are as in Fig. 6. Compared with model $\mathrm{B}_{\mathrm{T}}$ (Fig. 8 right column), we see more evidence of the original velocities of the clouds from the non-interacting parts, because of the change of perspective. This calculation produces the best fit to the observations (Fig. 2).

two velocity components, which would match the observations more closely.

\subsection{Timescales}

One interesting question for the star-formation history of Serpens is to confirm whether or not the older population of premain sequence star of $2 \mathrm{Myr}$ of age could have been formed in the same cloud-cloud collision event. For this we need to estimate a number of characteristic timescales. First, all the simulations correspond to a total elapsed time of $\sim 8-9 \times 10^{5}$ years. However, it is only after about $2-3 \times 10^{5}$ years, about a third of the total simulation time, that the two cylinders begin to interact. This time delay is needed, especially in the turbulent cases, to allow the cylinders to relax their initial density distributions. The time from the start of the interaction between the two clouds until they begin to form sink particles is of the order 


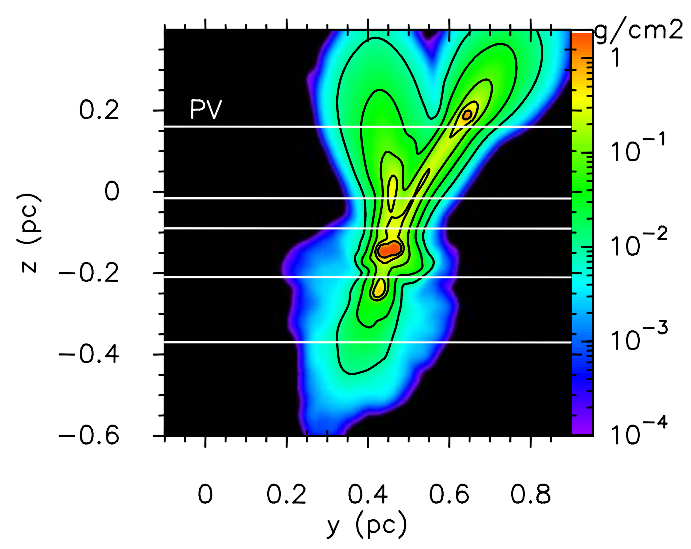

Fig. 11. Total column density for the non-turbulent, purely along the line-of-sight run with longer cylinders, $\mathrm{D}_{\text {non-T }}$, to test the production of both the NW and SE sub-clusters of Serpens. At this stage, sink particles have indeed formed where the collision is happening and in the north-west (within the tilted/longer cylinder). Given the geometry of the collision, the two cylinders do not end up colliding in the north. However, the only perspective where we do not see the cylinders overlap is the one we took for this run, where the line of sight is aligned with the motion of the cylinders. The contour levels are as in Fig. 5.

of $6 \times 10^{5}$ years. For the 2 Myr stellar population to be able to form through this collision, the duration of the interaction time has to be at least 2 Myr. In fact, we would need the cloud to be one order of magnitude larger (i.e. a few parsec wide) in order to still have an ongoing collision $2 \mathrm{Myr}$ after the formation of the first protostars. This seems unrealistic and therefore, the cloud collision scenario we present for Serpens is consistent with the idea of two separate bursts of star formation in the region.

\section{Discussion}

Overall the simulation that best represents the SE sub-cluster of the Serpens star-forming region is model $\mathrm{C}_{\mathrm{T}}$, the offset turbulent model with short cylinders, reprojected so that the line-ofsight coincides with the direction of motion. It has a velocity structure similar to the observed one, both comparing the PV diagrams and the general trend in the velocity coded 3-colour plots (Figs. 13 and 14). Note that this simulation only represents the southern part of Serpens, because the NW sub-cluster is not directly involved in the collision (cf. Sect. 4.1). Despite this, the 3-colour plot of this run (Fig. 14) agrees well with that of the observations (Fig. 13). Model $\mathrm{C}_{\mathrm{T}}$ shows an overall velocity gradient of $\sim 2 \mathrm{~km} \mathrm{~s}^{-1}$ over $0.2 \mathrm{pc}^{-1}$, from blue in eastern regions to red in the west (Fig. 14), similar to what is observed.

The 3-colour velocity figures also show the resemblance of the simulation $\mathrm{C}_{\mathrm{T}}$ with the observations in the way that the filament extending south is mostly represented by the red/green velocities, while the blue part is mainly seen on the denser parts where the stars are being formed. The green-blue component is also seen farther east, forming some less dense filaments perpendicular to the main filament. Note that we do not see these types of filament on the red side.

Understanding the nature of these filaments can be important. If these filaments had been caused by the initial turbulence, we would expect them to exist in both directions. Alternatively, these filaments could be the consequence of some particles left behind as the cylinders move, resembling a tail. However, in simulation $\mathrm{C}$, the cylinders' motion is along the line of sight, so if these filaments resulted from a tail of material left behind during the collision, they would be behind or in front of the main

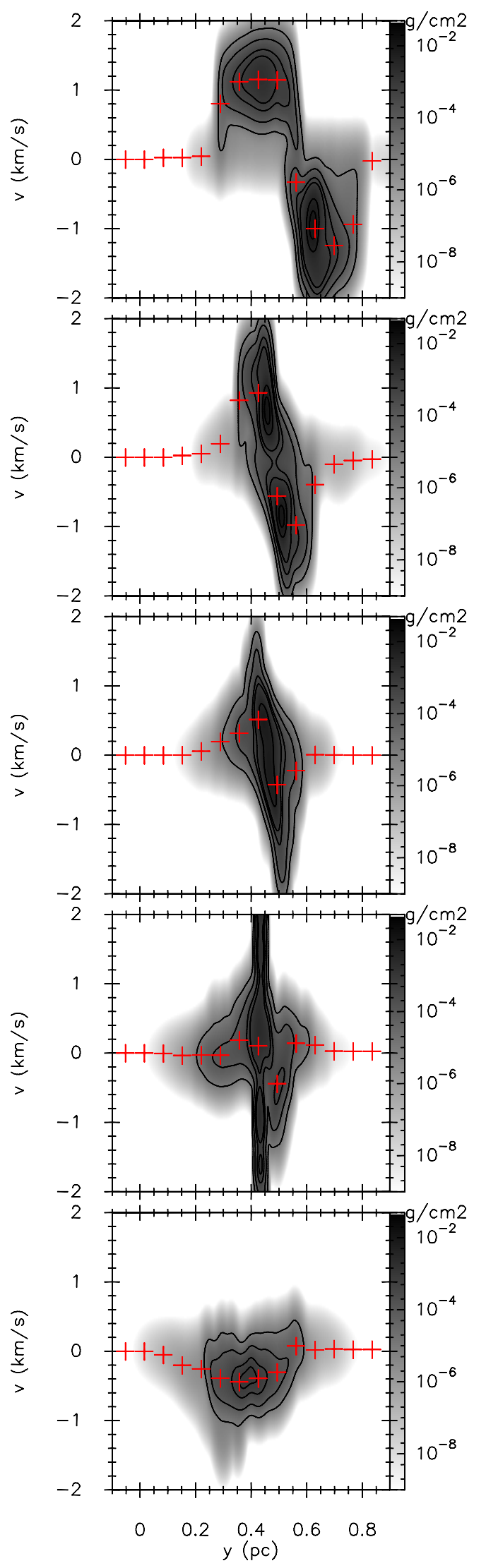

Fig. 12. $\mathrm{PV}$ diagrams of the long cylinder run $\mathrm{D}_{\text {non-T }}$ at $z=0.16 \mathrm{pc}$, $z=-0.02 \mathrm{pc}, z=-0.09 \mathrm{pc}, z=-0.21 \mathrm{pc}$ and $z=-0.37 \mathrm{pc}$ (from top to bottom). Contour levels and red crosses are as in Fig. 6. Despite the lack of structure typical of a non-turbulent case, these agree with the more detailed PV diagrams from observations from Duarte-Cabral et al. (2010) and Graves et al. (2010). 
A. Duarte-Cabral et al.: Was a cloud-cloud collision the trigger of the recent star formation in Serpens?

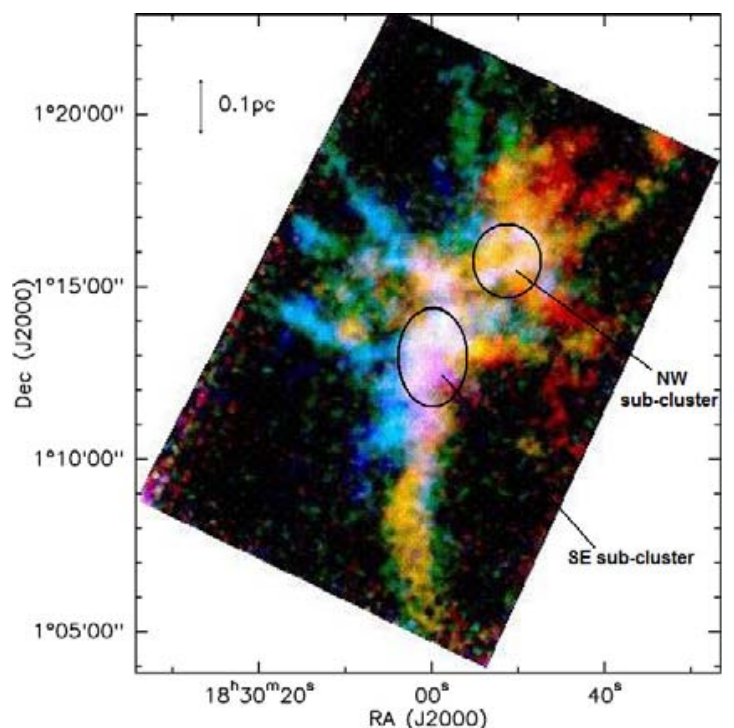

Fig. 13. Velocity-coded 3-colour plot of Serpens from the $\mathrm{C}^{18} \mathrm{O} J=$ $3 \rightarrow 2$ data from the JCMT GBS. Each colour represents the maximum value in the velocity intervals: blue: $5 \rightarrow 7.7 \mathrm{~km} \mathrm{~s}^{-1}$; green: $7.7 \rightarrow$ $8.3 \mathrm{~km} \mathrm{~s}^{-1}$; and red: $8.3 \rightarrow 11 \mathrm{~km} \mathrm{~s}^{-1}$ (see also Graves et al. 2010). The two sub-clusters of Serpens are indicated by the black ellipses.

structure. Their appearance therefore indicates that these perpendicular filaments are caused by the geometry of the collision, in particular, its asymmetric nature. These filaments place a strong constraint on the geometry of the collision. The geometry of simulation A (as well as other tests not shown) do not reproduce them.

In terms of its total column density distribution, $22 M_{\odot}$ in $0.02 \mathrm{pc}^{2}$, model $\mathrm{C}$ closely matches the SE sub-cluster. Even the projected distribution of material above a column density of $0.1 \mathrm{~g} \mathrm{~cm}^{-2}$ resembles the shape of the SE sub-cluster.

To reproduce the NW sub-cluster in addition to the SE subcluster requires the longer non-turbulent clouds used in model $\mathrm{D}_{\text {non-T. }}$. In this model a region close to being gravitationally unstable can be perturbed and its collapse hastened by the collision, even though it is not directly involved in the collision. This second sub-cluster forms with the velocity of its native cloud and collapses smoothly and independently of the southern region. If this model is allowed to continue, the NW sub-cluster falls onto the SE sub-cluster. Intriguingly, recent observations of the magnetic field in Serpens (Sugitani et al. 2010) appear to suggest the start of such a collapse, which could result in the merging of the two sub-clusters.

\section{Conclusions}

Serpens is a very interesting star-forming region, not only for its youth, but also for the striking differences between the two sub-clusters that compose the active star-forming portion of the cloud. Even though they are at similar stages of evolution, with most sources between Class 0 and Class I protostars and similar dust continuum properties, the gas emission reveals that they have not only different velocity characteristics, but also different temperature distributions (Duarte-Cabral et al. 2010).

Motivated by the two velocity components visible in the southern sub-cluster of Serpens and by the higher temperatures detected there, we performed several SPH calculations of cloudcloud collisions. The configurations used for these simulations were based on the observed morphologies.

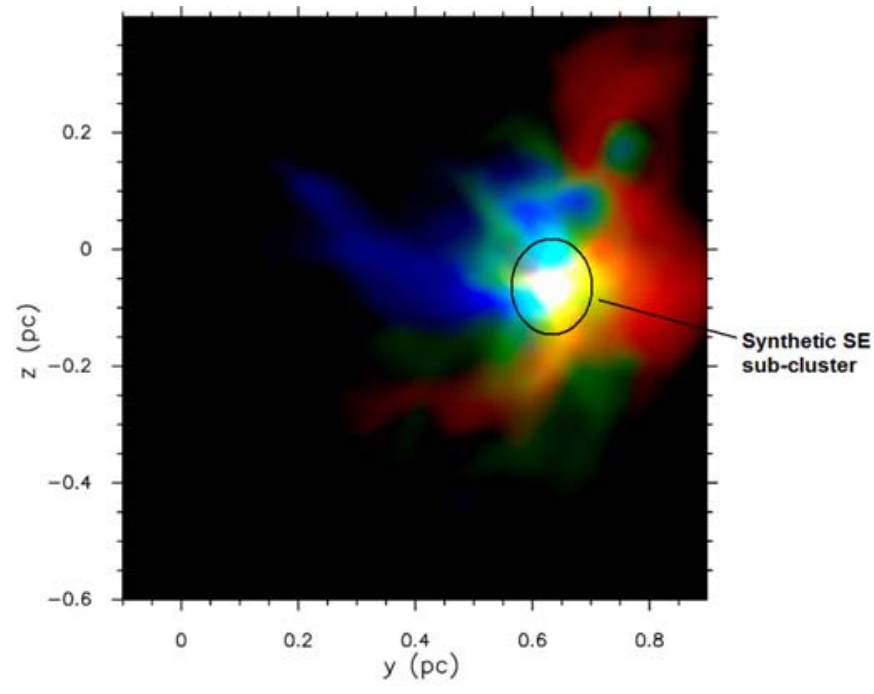

Fig. 14. Velocity-coded 3-colour plot of the simulation $C_{T}$. Each colour represents the total column density summed over the following line of sight velocities: blue: $0.3 \rightarrow 2 \mathrm{~km} \mathrm{~s}^{-1}$; green: $-0.3 \rightarrow 0.3 \mathrm{~km} \mathrm{~s}^{-1}$; and red: $-2 \rightarrow-0.3 \mathrm{~km} \mathrm{~s}^{-1}$. This figure is to be compared with Fig. 13, particularly around the SE sub-cluster. The synthetic SE sub-cluster is indicated by a black ellipse.

For the SE sub-cluster, a model of two colliding clouds is able to reproduce the column density structure, a centrally condensated filament aligned in a NW-SE direction, and the two velocity components visible where the star formation driven by the collision is occurring. However, the same simulations did not produce a second sub-cluster, similar to the NW sub-cluster of Serpens. Therefore, this sub-cluster does not seem to be the direct result of the collision. This was already suggested by the NW sub-cluster's well "behaved" temperature profile and velocity structure, as well as by the uniform age of sources within the sub-cluster. Nevertheless, the similar stage of evolution of the sources from the two sub-clusters and their proximity suggests that the two events are not totally independent.

A simulation with more elongated cylinders and increased masses provides a possible explanation. The presence of a marginally stable region in the northern part of one of the colliding clouds can have its collapse induced and quickened by perturbations driven by the cloud-cloud collision.

We consider a cloud-cloud collision scenario to be the best description of the driving of the star-formation history in Serpens. Not only can it reproduce the observed velocities and column densities, it also offers a plausible explanation for why the two sub-clusters are so similar in some aspects and yet so different in others. Although cloud rotation may produce similar general velocity gradients to those observed, the complexity of the region is better explained with such a collision scenario, which is in essence similar to a shear-motion also suggested by Olmi \& Testi (2002).

Despite the successful scenario provided by a cloud-cloud collision model, we failed to reproduce all of the Serpens characteristics in one single run. Additional support against gravity is required in order to sustain the existence of two different sub-clusters as in Serpens. The existing magnetic field of the region (Sugitani et al. 2010) could provide this support.

Acknowledgements. Ana Duarte Cabral is funded by the Fundação para a Ciência e a Tecnologia of Portugal, under the grant reference SFRH/BD/36692/2007. A.D.C. would like to thank Jennifer Hatchell for useful discussions and suggestions that initially motivated this work. The 

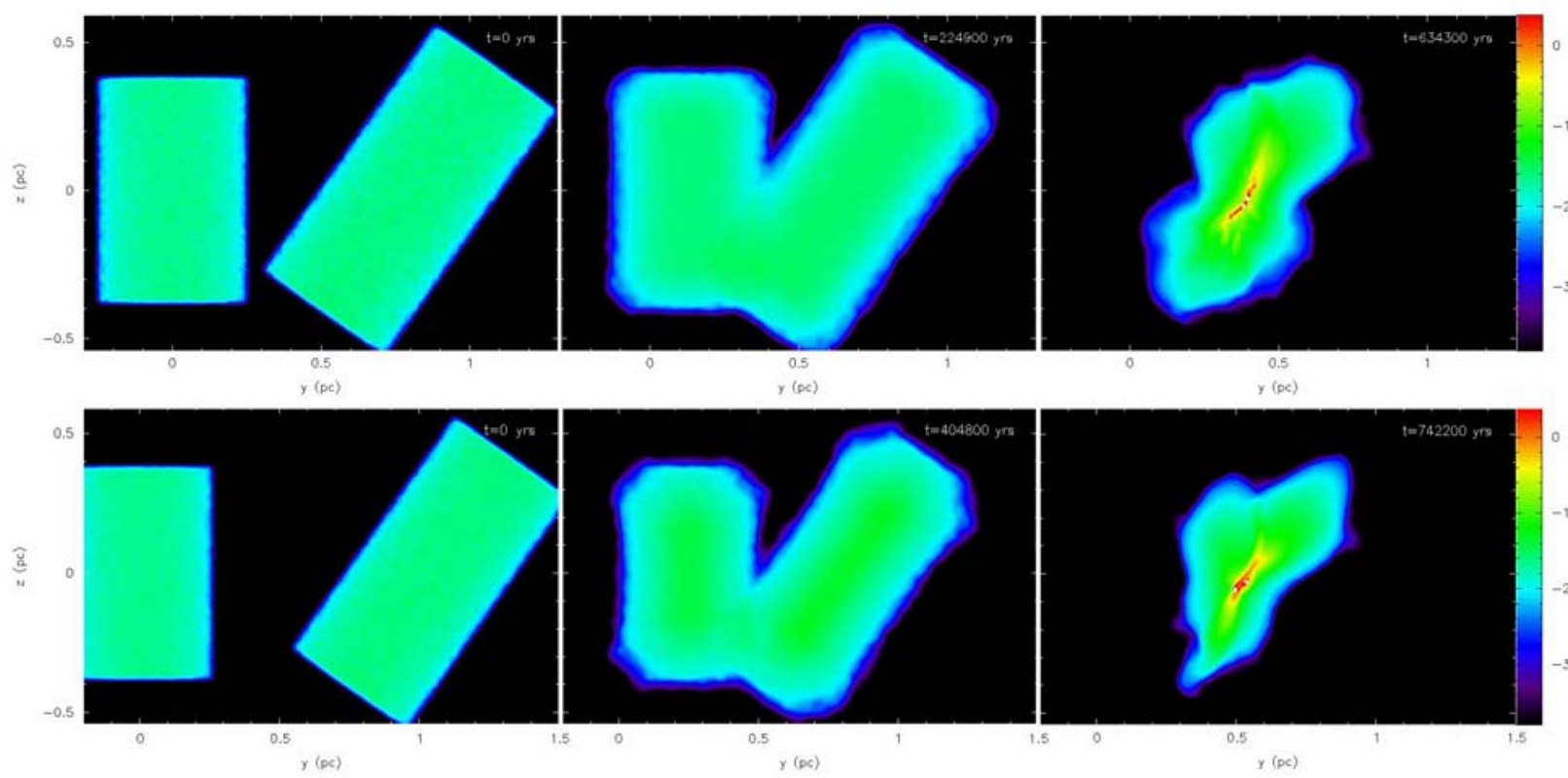

Fig. A.1. Three time snapshots of the total column density along the line of sight for the non-turbulent runs: the centred collision, $A_{\text {non-T }}(t o p)$ and off-centred collision, $\mathrm{B}_{\text {non-T }}$ (bottom). The three frames correspond to the beginning of the simulation (first frame), as soon as the cylinders start to collide (second frame) and when the first sink particle is formed (last frame).
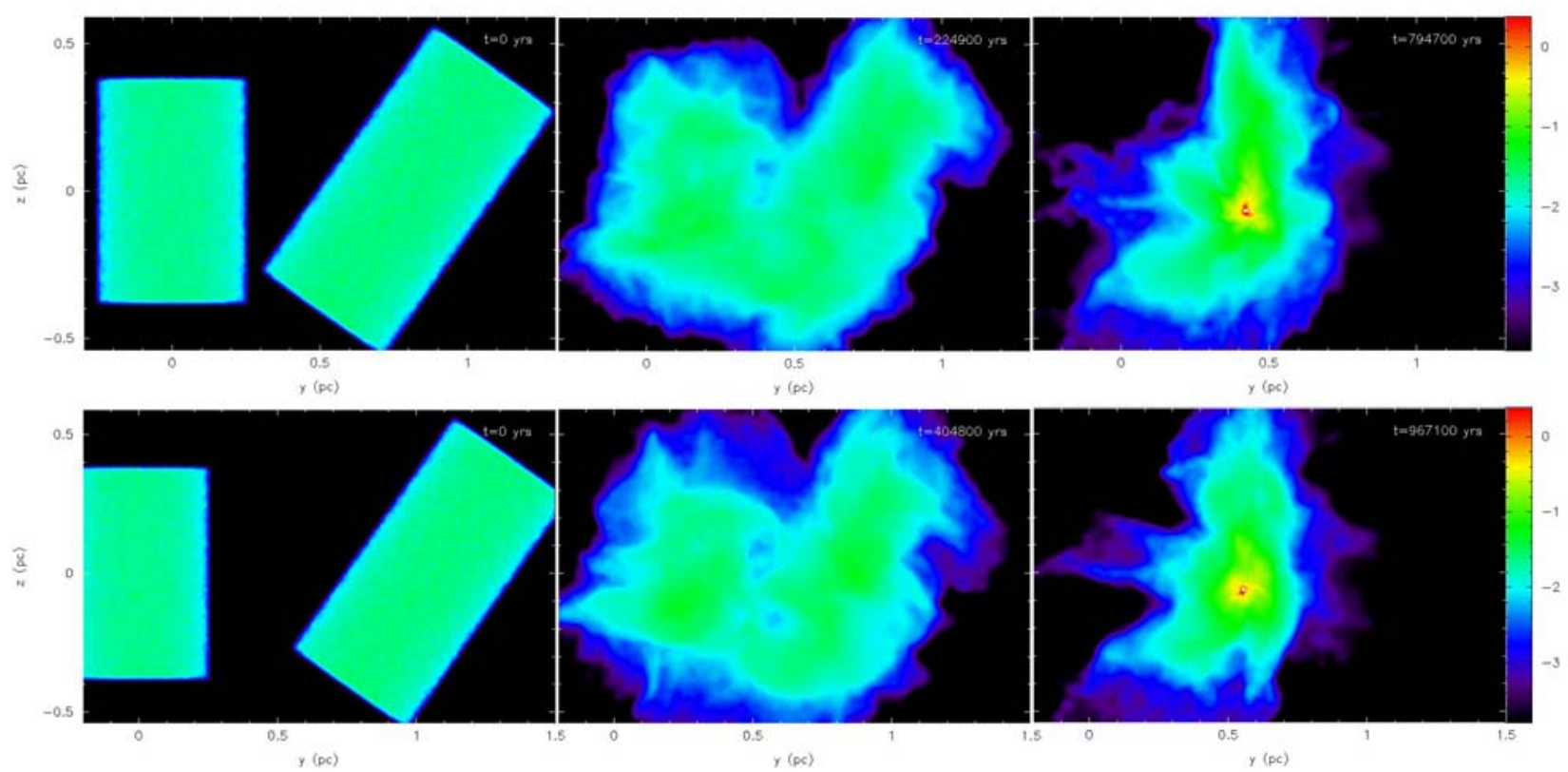

Fig. A.2. Three time snapshots of the total column density along the line of sight for the turbulent runs: the centred collision, $\mathrm{A}_{\mathrm{T}}$ (top) and offcentred collision, $\mathrm{B}_{\mathrm{T}}$ (bottom). The three frames correspond to the beginning of the simulation (first frame), as soon as the cylinders start to collide (second frame) and when the first sink particle is formed (last frame).

calculations reported here were performed using the University of Exeter's SGI Altix ICE 8200 supercomputer. C.L.D.'s work was conducted as part of the award "The formation of stars and planets: radiation hydrodynamical and magnetohydrodynamical simulations" made under the European Heads of Research Councils and European Science Foundation EURYI (European Young Investigator) Awards scheme and supported by funds from the Participating Organisations of EURYI and the EC Sixth Framework Programme.

\section{Appendix A: Time snapshots of the models}

Figures A.1 to A.4 show three snapshots to illustrate the time evolution of the models. Each frame is a projected column density plot as seen from the chosen line of sight. The time (in years) is shown in the top-right corner of each frame. Figure A.1 shows three frames for the two non-turbulent runs, $A_{\text {non-T }}$ and

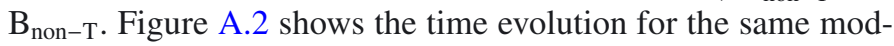
els, A and B, but with turbulence. Figure A.3 shows the snapshots of model $\mathrm{C}$, which is essentially the same as model $\mathrm{B}_{\mathrm{T}}$, only with a different line of sight. For these three runs, the last frame corresponds to the formation of the first sink particle and is the frame used to construct a datacube for comparison with the observations. Finally, Fig. A.4 shows three snapshots for run D. Note that for this run we are showing frames after the formation 

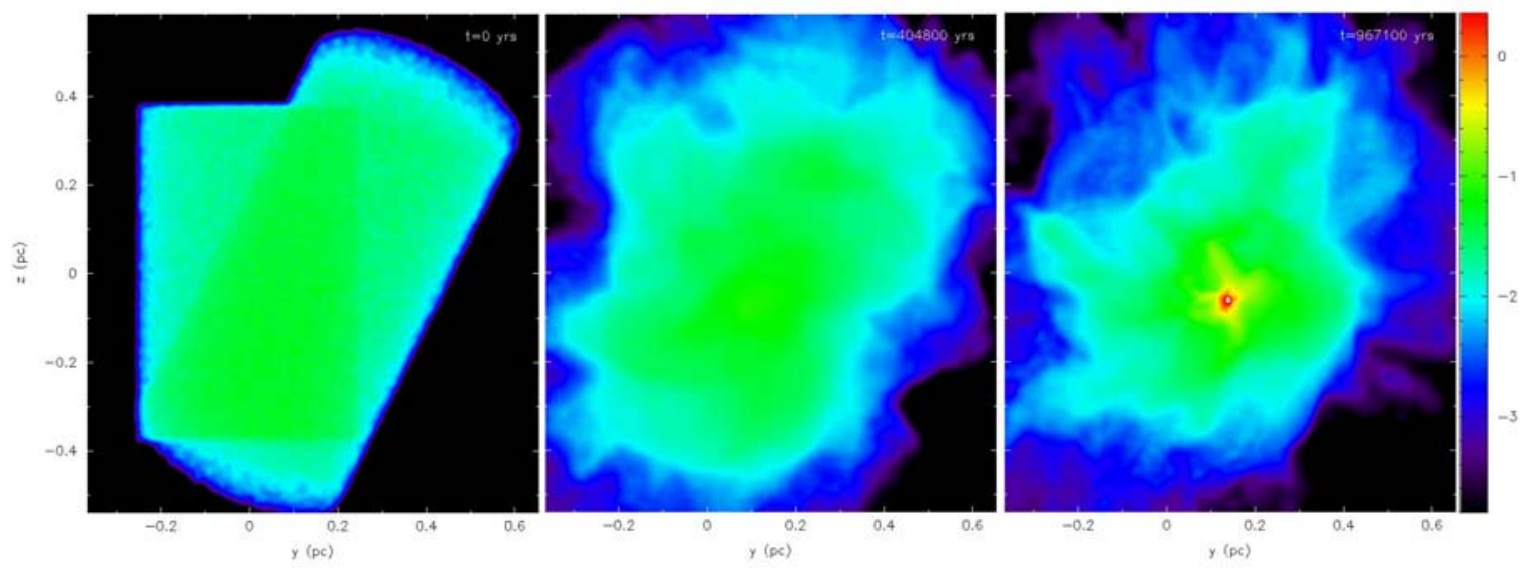

Fig. A.3. Three time snapshots of the total column density along the line of sight for $\mathrm{C}_{\mathrm{T}}$. The three frames are as before. Note that this is exactly the same model as $\mathrm{B}_{\mathrm{T}}$, but viewed from a different perspective.
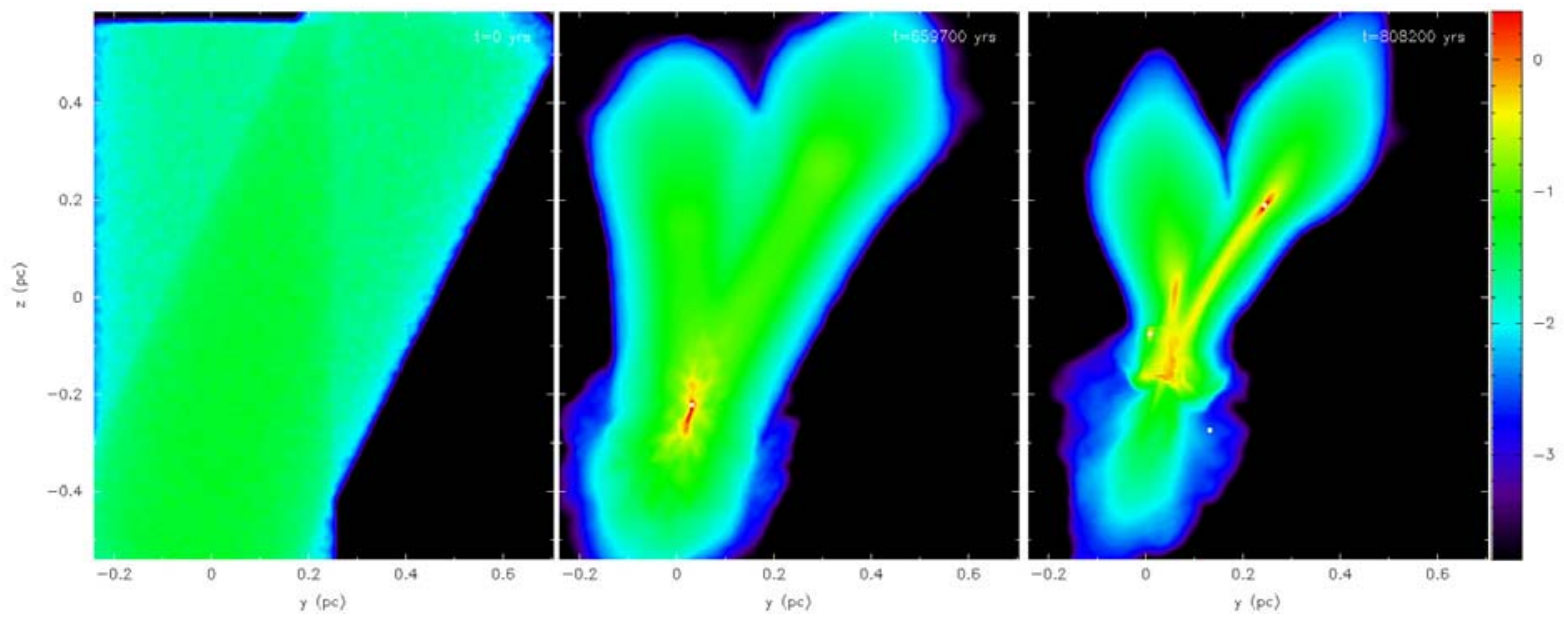

Fig. A.4. Three time snapshots of the total column density along the line of sight for $\mathrm{D}_{\text {non-T. }}$. The three frames are at the beginning of the simulation (left), when the first sink particle forms in the south (middle) and when a sink particle is formed in the north (right).

of the first sink particle, to demonstrate where and when the second clump in the north is able to form a sink particle. In this case, this last frame was the one used for the comparison with the observations.

\section{References}

Anathpindika, S. 2009a, A\&A, 504, 451

Anathpindika, S. 2009b, A\&A, 504, 437

André, P., Men'shchikov, A., Bontemps, S., et al. 2010, A\&A, 518, L102

Ballesteros-Paredes, J., Hartmann, L., \& Vázquez-Semadeni, E. 1999, ApJ, 527, 285

Bastien, P. 1983, A\&A, 119, 109

Bate, M. 1995, Ph.D. Thesis, Univ. Cambridge

Bate, M. R. 2009a, MNRAS, 392, 590

Bate, M. R. 2009b, MNRAS, 397, 232

Bate, M. R., Bonnell, I. A., \& Bromm, V. 2002, MNRAS, 332, L65

Bate, M. R., Bonnell, I. A., \& Bromm, V. 2003, MNRAS, 339, 577

Benz, W., Cameron, A. G. W., Press, W. H., \& Bowers, R. L. 1990, ApJ, 348, 647

Dale, J. E., Bonnell, I. A., \& Whitworth, A. P. 2007, MNRAS, 375, 1291

Dale, J. E., Wünsch, R., Whitworth, A., \& Palouš, J. 2009, MNRAS, 398, 1537

Davis, C. J., Matthews, H. E., Ray, T. P., Dent, W. R. F., \& Richer, J. S. 1999, MNRAS, 309, 141

Dobbs, C. L. 2008, MNRAS, 391, 844

Dobbs, C. L., Bonnell, I. A., \& Pringle, J. E. 2006, MNRAS, 371, 1663 Duarte-Cabral, A., Fuller, G. A., Peretto, N., et al. 2010, A\&A, 519, A27

Dubinski, J., Narayan, R., \& Phillips, T. G. 1995, ApJ, 448, 226

Elmegreen, B. G., \& Lada, C. J. 1977, ApJ, 214, 725
Galván-Madrid, R., Zhang, Q., Keto, E., et al. 2010, ApJ, 725, 17 Gittins, D. M., Clarke, C. J., \& Bate, M. R. 2003, MNRAS, 340, 841 Graves, S. F., Richer, J. S., Buckle, J. V., et al. 2010, MNRAS, 409, 1412 Hartmann, L., \& Burkert, A. 2007, ApJ, 654, 988

Harvey, P. M., Chapman, N., Lai, S.-P., et al. 2006, ApJ, 644, 307

Harvey, P. M., Rebull, L. M., Brooke, T., et al. 2007, ApJ, 663, 1139

Heitsch, F., Hartmann, L. W., Slyz, A. D., Devriendt, J. E. G., \& Burkert, A. 2008, ApJ, 674, 316

Heitsch, F., Ballesteros-Paredes, J., \& Hartmann, L. 2009, ApJ, 704, 1735

Higuchi, A. E., Kurono, Y., Saito, M., \& Kawabe, R. 2010, ApJ, 719, 1813

Kaas, A. A., Olofsson, G., Bontemps, S., et al. 2004, A\&A, 421, 623

Kitsionas, S., \& Whitworth, A. P. 2007, MNRAS, 378, 507

Klessen, R. S., Ballesteros-Paredes, J., Vázquez-Semadeni, E., \& Durán-Rojas, C. 2005, ApJ, 620, 786

Koda, J., Sawada, T., Hasegawa, T., \& Scoville, N. Z. 2006, ApJ, 638, 191

Larson, R. B. 1981, MNRAS, 194, 809

Molinari, S., Swinyard, B., Bally, J., et al. 2010, A\&A, 518, L100

Myers, P. C. 2009, ApJ, 700, 1609

Olmi, L., \& Testi, L. 2002, A\&A, 392, 1053

Oort, J. H. 1954, Bull. Astron. Inst. Netherlands, 12, 177

Palla, F., \& Stahler, S. W. 2002, ApJ, 581, 1194

Peretto, N., Hennebelle, P., \& André, P. 2007, A\&A, 464, 983

Price, D. J., \& Monaghan, J. J. 2005, MNRAS, 364, 384

Price, D. J., \& Bate, M. R. 2007, MNRAS, 377, 77

Schneider, N., Csengeri, T., Bontemps, S., et al. 2010, A\&A, 520, A49 Scoville, N. Z., Sanders, D. B., \& Clemens, D. P. 1986, ApJ, 310, L77

Straižys, V., Černis, K., \& Bartašiūte, S. 1996, Baltic Astron., 5, 125

Sugitani, K., Nakamura, F., Tamura, M., et al. 2010, ApJ, 716, 299

Tasker, E. J., \& Tan, J. C. 2009, ApJ, 700, 358

Vallee, J. P. 1995, AJ, 110, 2256 